\title{
Treatment of Hydrocele Testis with Aspiration and Sclerotherapy
}

\author{
Kim Drasa Jr, Evin Dani \\ Central Polyclinic, Tirana, Albania
}

\begin{abstract}
Introduction and Objective: Acquired hydrocele testis is a common cause of increase scrotal size in the adult male. In this study our aims is to demonstrate that Hydrocele aspiration sclerotherapy (HAS) with doxycycline is an effective and safe nonsurgical treatment option for the nonseptated hydrocele correction. Material and Methods: HAS was performed in an outpatient office setting in all patients in this study. Before undergoing HAS all patients were evaluated with a history, a thorough genital examination, and scrotal ultrasound to determine the nature of hydrocele. Men who presented with a nonseptated hydrocele and were interested in nonsurgical correction were eligible for HAS. Exclusion criteria were presentation with a multiseptated hydrocele, a recidivant hydrocele, spermatocele, infection or hernia. Results: There were 18 patients with mean age of 51.3 years who presented with nonseptated hydrocele testis between 2008- 2013. Overall mean follow-up was 22.2 months. Of these hydroceles $83.3 \%(n=15)$ were successfully treated with a single HAS procedure. In the group reporting success 13 patients presented with unilateral hydrocele and 2 presented with a bilateral hydrocele. Mean, median and range of fluid aspirated from successful treatment group were 195cc (SD 115.3) and 100 to $500 \mathrm{cc}$, respectively. Of those patients in whom HAS failed,one had hydrocele successfully with a second HAS treatment,2 did not have success with a second HAS and underwent hydrocelectomy. Conclusion: HAS appears to be a safe quick, far less costly and reasonably effective in-office procedure for the treatment of nonseptated single hydroceles. Our findings demonstrate that success rate with HAS are similar to those with surgical management while avoiding many of the complications and expenses associated with surgery. For patients in whom HAS failes, surgical hydrocelectomy remains a viable treatment options.
\end{abstract}

Keywords: Testicular hydrocele, Sclerotherapy, doxocyclin.

\section{Introduction}

A description of hydroceles dates to Galen in 176 AD.A change in scrotal size can be a disturbing physical change for any adult male. Scrotal pain during intercourse of physical activity, discomfort related to the enlarged scrotum, cosmetic appierants of the scrotum, concerns of the damage to the reproductive organs or possible malignancy are reasons why men sick evaluation for any increase in scrotal size.

The diagnosis and management of scrotal pain are common components of urological office practice and most scrotal surgery is considered routine, often the intern's case. The answer is that scrotal pathology is common and, as such, provides an opportunity for innovation in treatment and potentially significant savings in costs.

Acquired hydrocele is a common cause of increased scrotal size in the adult male which affect approximately $1 \%$ of men and is mostly seen after age 40 years[1].The conventional treatment of a symptomatic hydrocele is surgical [2] and hydrocelectomy continuous to be the most common method of treatment.Complications scrotal of this benign conditions include prolonged pain, recurrence, hematoma, infection and injury to the scrotal contents including the testicle epididymis and /or vas deferns[3]. Furthermore, surgical management is associated with substantial cost including absence from work for recovery and the use of hospital resources.

Hydrocele aspiration and sclerotherapy was first reported in 1975 as a nonsurgical outpatients treatment for hydroceles [4]. Sclerotherapy for asymptomatic hydrocele testis has been increasingly used and advocated as a minimally invasive procedures, and a variety of sclerosing agent have produced different outcomes[4].
Numerous chemical and pharmacological agents can be used as sclerotherapy agents with varied success, including $99.5 \%$ alcohol [5], ethanolamineoleate [6], sodium tetradecylsulfate [7]- [8], tetracycline [9]- [10] and doxycycline (an inexpensive tetracycline derivative).

The therapeutic efficacy of these agents varies considerably [11].The best effect $(45 \%$ to $85 \%)$ cure rate has been reported for doxycycline or polidocanol, whereas the corresponding figures for sodium tetradecylsulphate, phenol and other sclerosants range from $36 \%$ to $52 \%$.

Complications oftreatment included pain, hematoma and epididymitis. However, when treatment is indicated, hydrocele testis has often been managed with open hydrocelectomy, which is considered the gold standard [12]. In the treatment of hydroceles, aspiration and sclerorotherapy are regarded as a minimally invaside approach that is simple, inexpensive and safe, but less effective than hydrocelectomy, as shown in a nonrandomised study [13]. Currently to our knowledge there are one published reports of HAS using doxacycline as a sclerosing agent. Therefore in this retrospective analysis we will demonstrate that HAS with doxycycline in an effective and safe nonsurgical treatment option for nonseptated hydrocele.

\section{Materials \& Methods}

\section{Patients Selection}

Men with symptomatic hydrocele testis during 2008 to 2013 were consecutively included in this prospective randomised, double- blind study. Aspiration of sclerotherapy were performed at outpatient centralpolyclinic in Tirana, Albania (where we work), in all patients of our study. Before undergoing treatment were taken the patients histories. All 


\section{International Journal of Science and Research (IJSR) \\ ISSN (Online): 2319-7064}

Index Copernicus Value (2013): 6.14 | Impact Factor (2014): 5.611

the patients underwent a thorough genital examination and scrotal Doppler ultrasound to determine the nature of the hydrocele and to rule out other scrotal abnormalities including tumor, spermatocele, multiseptated hydrocele, infection or hernia. Careful oral and written instructions were given. Patients were made aware of the risk and the benefits of HAS, as well as the option for surgical management, and all patients underwent an informed consent process. After having sign informed consent, the patients were randomised to active treatment at the first treatment following the study design.

\section{Aspiration and Sclerosing Procedure}

The HAS procedure has been describedelsewhere. The procedure for HAS wasa) Cleansed the side of the affected scrotum with betadine, b) Unilateral spermatic cord block with $10 \mathrm{cc}-0.5 \%$ bupivacaine,c) A 12 gauge angiocatheter was inserted percutaneously into the hydrocele sac on the anterior superior or lateral side of the scrotum, d)the hydrocele fluid was aspirated using a 60cc syringe with extension tubing and stopcock depending on the size of the hydrocele (less than 100,100 to 200 and greater than 200), e) An injection through the angiocatheter into the empty hydrocele space of 200 to $400 \mathrm{mg}$ doxycycline diluted in 10 cc- $0.5 \%$ bupivacaine without epinephrine, f) The scrotum was massaged for two minute to expose the lining of the hydrocele sac to the sclerosing agent. The procedure typically took 20-30 minutes to complete. Patients were given NSAD for post-operative pain. The $400 \mathrm{mg}$ dose of doxycycline was generally reserved for patients with hydroceles larger than 500cc in volume. After removal of the needle a skin patch was placed on the scrotal skin. Patients were given oral and written information on when and how to take painkillers if necessary to manage postoperative pain. Patients were asked to note normalization of scrotal size and any improvement in pain or discomfort. No antibiotics were used. After treatment the patients were asked to perform a scrotal massage for two minutes on the following days when they went to the bathroom to spread the sclerosing agent and for a better result, with otherwise norestrictions. Patients were instructed to follow-up in the office between 6 and 8 weeks after the procedure for the reassessment.

\section{Data Collection}

Study data were collected by review of participant medical records. At the initial follow- up, patients were stratified into success and failure groups based on subjective and objective measures of treatment outcome. Patients whom reported outcomes were gathered using a nonvalidated questionnaire addressing post treatment pain, resolution or persistent of the hydrocele, other symptoms associated with hydrocele and satisfaction with the procedure. A successful HAS procedure was defined as complete to minimal persistence of hydrocele (less than $30 \mathrm{cc}$ ), improvement in symptoms of pain or discomfort. Criteria for failure included recurrence of the hydrocele and no improvement in pain,discomfort, activities of daily leaving or presentation of hydrocelectomy.At initial follow-up patients in whom a single HAS procedure failed were presented with the 3 options: observation, repeat HAS or surgical hydrocelectomy.

\section{Results}

There were 18 patients with mean age of 51.3 years who presented with nonseptated hydrocele testis between 2008 2013. Overall mean follow-up was 22.2 months. Of these hydroceles $83.3 \%(\mathrm{~N}=15)$ were successfully treated with a single HAS procedure. In the group reporting success 13 patients presented with unilateral hydrocele and 2 patients presented with a bilateral hydrocele. Mean, median and range of fluid aspirated from successful treatment group were $195 \mathrm{cc}$ (SD 115.3) and 100 to $500 \mathrm{cc}$, respectively. Of those patients in whom HAS failed, one had hydrocele successfully with a second HAS treatment, 2 did not have success with a second HAS and underwent hydrocelectomy. The origin of the hydrocele was believed to be idiopathic in 1 of these 3 patients whom HAS failed. The other 2 patients whom underwenthydrocelectomy reported that hydroceles occurred after varicocelectomy.

The main side effects with HAS procedure included a moderate amount pain, reported $5(27.8 \%)$ patients, which resolved in 2 to 3 days. One patient reported severe transient epigastric pain and another had a vasovagal reaction during the procedure. Three patients reported a pulling sensation in the scrotum that eventuality resolved. No patients required hospital administration.

\section{Discussion}

Acquired hydroceles may form as reaction to tumours, infections or trauma, but most are indiopathic in origin. The pathophysiology of an acquired hydrocele testis is unclear, but may result from increased serous fluid secretion, lack of efferent lymphatic's of failure of lymphatics in the mesothelial lining to reabsorb fluid [14]. The hydrostatic pressure of hydrocele has been demonstrated to be greater than the pressure of the blood vessels in the scrotum, which may create stasis in venous and lymphatic flow which results in an accumulation of fluid with an increase in scrotal size [15].

In a previous study by Yilmaz et al. comparing hydrocele therapy with aspiration alone versus aspiration and sclerotherapy, the hydrocele recurred in all patients treated with aspiration alone [16]. Comparing the recurrence rate after aspiration alone to aspiration and sclerotherapy with doxycycline may serve as an active control in a future trial and provide insight into the efficacy of the sclerotherapy component of the procedure. It appears thatsclerotherapy is neccessary after aspiration to create the inflammatory response and subsequent fibrosis which impede the flow of fluid into the hydrocele sac, thereby more effectively preventing recurrence. Tetracycline was previously used as a sclerosing agent for HAS but has been replaced by doxycycline because the injectable formulation of tetracycline is no longer available in United States.

Prof. Dr. Maksut Drasa (my father) has been the best General surgeon, Urologists, in Balkan (1960-1985) and in his study before 40 years ago, had used Tetracycline for HAS in his study (17). Based on the conclusion of this study he let me a 


\section{International Journal of Science and Research (IJSR) \\ ISSN (Online): 2319-7064 \\ Index Copernicus Value (2013): 6.14 | Impact Factor (2014): 5.611}

very important advice: “My son, in your practice don't use the tetracycline for sclerotization of hydrocele testis after aspiration because may give shock (vasovagal reaction) during installation of tetracycline."

\section{Conclusion}

We advocate aspiration and sclerotherapy for the treatment of hydrocele testis because it involves less morbidity, a lower complication rate and fewer costs than any current alternatives. Our findings demonstrate that success rates with HAS are similar to those with surgical management. For patients in whom the HAS fails, surgical hydrocelectomy remains a viable treatment option.

\section{References}

[1] Leung ML, Gooding GA and Williams BD: High resolution sonography of scrotal contents in asymptomatic subjects. AJR J Roentgenol 1984; 143: 161.

[2] Lord PH: A bloodless operation for the radical cure off idiopathic hydrocele. Br J Surg 1964; 51:914.

[3] Swartz MA, Morgan TM and Krieger JN: Complications of scrotal surgery for benign conditions. Urology 2007; 69: 616 .

[4] Moloney GE: Comparison of results of treatment of hydrocele and epididymal cysts by surgery and injection. Br Med J 1975; 3: 478.

[5] Shan CJ, Lucon AM, Pagani R et al: Sclerotherapy of hydroceles and spermatoceles with alcohol: results and effects on the semen analysis. IntBraz J Urol 2011; 37: 307.

[6] Tammela TL, Hellstrom PA, Mattila SI et al: Ethanolamine oleatesclerotherapy for hydroceles and spermatoceles: a survey of 158 patients with ultrasound followup. J Urol 1992; 147: 1551.

[7] Beiko DT, Kim D and Morales A: Aspiration and sclerotherapyvshydrocelectomy for treatment of hydroceles. Urology 2003; 61: 708.

[8] Rencken RK, Bornman MS, Reif MS et al: Sclerotherapy for hydroceles. J Urol 1990; 143: 940.

[9] Daehlin L, Tonder B and Kapstad L: Comparison of polidocanol and tetracycline in the sclerotherapy of testicular hydrocele and epididymal cyst. $\mathrm{Br} \mathrm{J}$ Urol 1997; 80: 468.

[10] ShokeirAA, Erkay I, Hassan N et al: Tetracycline sclerotherapy for testicular hydroceles in transplant recipients. Urology 1994; 44: 96.

[11] Sigurdsson T, Johansson JE, Jahnson $\mathrm{S}$ et al: Polidocanolsclerotherapy for hydroceles and epididymal cysts. J Urol 1994; 151: 898.

[12] Epstein A and Novicki DE: Management of hydroceles. AUA Update Series 1988; 7: lesson 19, p 145.

[13]Beiko DT, Kim D and Morales A: Aspiration and sclerotherapyvs hydroceles. Urology 2003; 61: 708.

[14] Mihmanli I, Kantarci F, Kulaksizoglu h et al: Testicular size and vascular resistance before and after hydrocelectomy. AJR Am J Roentgenol 2004; 183: 1379.
[15] Yilmaz U, Ekmekcioglu O, Tatlisen A et al: Does pleurodesis for pleural effusions give bright ideas about the agents for hydrocele sclerotherapy? IntUrolNephrol 2000; 183: 1379.

[16]Drasa M: Hydrocele tunica vaginal testis. Textbook: "Surgical OperationTechniques"; 1984; 623: 440-445. 